\title{
Resilience, Emotional Stability and Perceived Stress among 10th graders
}

\author{
Shruti Chandelkar ${ }^{1}$, Vidyadayani Shetty ${ }^{2}$ \\ ${ }^{1}$ Student, Department of Psychology, Nagindas Khandwala College, Mumbai. \\ ${ }^{2}$ Associate Professor, Department of Psychology, Nagindas Khandwala College, Mumbai. \\ Corresponding author: Vidyadayani Shetty \\ Email: vidhi33@gmail.com
}

\begin{abstract}
Background: In a day to day life we come across many hassles in our life and these hassles sometimes becomes stressors. Adolescence is a period of transitional stage with physical and psychological development wherein they face many such challenges and stressors. It depends on the individual ability, cognition and frame of life which help them to cope with stressors. The present study explores the relationship between resilience, emotional stability and perceived stress between boys and girls.

Methodology: The participants 10th graders, boys and girls were administered three scales, Resilience scale by Wagnild and Young, Emotional stability scale by A. S. Patel and Perceived stress scale by Sheldon Cohen. The data was analyzed, using the $t$ test and correlational coefficient.

Results: A significant negative correlation was found between resilience and perceived stress $(r=-.123)$ and for emotional stability and perceived stress $(r=-0.206)$. A significant positive correlation was found between resilience and emotional stability $(r=0.104)$. The $t$ values indicate that there was no significant gender difference in resilience, emotional stability and perceived scores. The $t$ values obtained for resilience, emotional stability and perceived stress scores of boys and girls having both parents working or single parent working did not indicate any significant difference.

Conclusion: A significant negative correlation was found between resilience and perceived stress and for emotional stability and perceived stress. A significant positive correlation was found between resilience and emotional stability. There was no significant gender difference in the three variables and no difference in the three variables for boys and girls of single parent/both parents working.
\end{abstract}

Keywords: Resilience, Emotional stability, Perceived stress, Parents, adolescents.

(Paper received $-5^{\text {th }}$ March 2019, Peer review completed $-12^{\text {th }}$ May 2018)

(Accepted $-14^{\text {th }}$ May 2019)

\section{INTRODUCTION}

Resilience refers to positive outcomes or adaptations or the attainment of developmental milestones or competencies in the face of significant risk, adversity or stress. In 1984, Garmezy, Masten, and Tellegen operationalized resilience in one of their earlier projects as, "manifestations of competence in children despite exposure to stressful events" [1]. Resilience is an interactive concept that refers to a relative resistance to environmental risk experiences, or the overcoming of stress or adversity. As such, it differs from both social competence positive mental health. Resilience differs from traditional concepts of risk and protection in its focus on individual variations in response to comparable experiences. The term resilience is used to refer to the finding that some individuals have a relatively good psychological outcome despite suffering risk experiences that would be expected to bring about serious sequelae [2].

Stress is a part and parcel of human lifestyle. It can serve as a driving force in terms of obtaining results, but on the other hand, non-stop stress can act as a killer in terms of performance. It is a known fact that students are subjected to different kinds of stressors, such as the pressure of academics with an obligation to succeed, 
an uncertain future, and difficulties of integrating into the system. The World Health Organization (WHO) has estimated that stress-related disorders will be one of the leading causes of disability by the year 2020 [3]. Stress of any type is a major risk for mental illness. In adolescence mental illness symptoms are often considered normal stressor for youth. Fifty percent of the adolescent population experiences their first mental illness symptoms prior to 18 years of age. However, $85-90 \%$ of young people with mental health problems did not seek mental help services. 9 Female adolescents are more vulnerable for stress than males 4 due to varying hormonal changes and social developments.10 Moreover, depression disorder is more evident in female adolescents [4]. Perceived stress occurs when an individual demonstrates a feeling of helplessness to control the stressful situation or to deal with the resulting emotional response. Therefore, according to the transactional model which emphasized that stress is essentially an interaction between the environment and the individual, the role of the individual's appraisal of the stressful events is vital in shaping his responses toward the perceived stress [5]. Further research on perceived stress and life events is desirable to promote a healthy lifestyle especially in the presence and stressors of a chronic disease in adolescence. Risk behaviours in adolescence, such as smoking and alcohol use, are associated with high levels of stress. These risk behaviours can be detrimental to the healthy adolescent and can be even worse on an adolescent who has cystic fibrosis. Exposure to smoke and alcohol can have adverse lung and gastrointestinal effects, which are already compromised in this population. Teaching effective stress-coping mechanisms early on can be a lifelong benefit [6].

One of the significant findings of the study was that perceived stress and resilience had relationship with life satisfaction in students. Most resilient boys and girls grew up in families with four or fewer children, with a space of two years or more between themselves and their next sibling. Few had experienced prolonged separation from a primary caretaker during the first year of life. The resilient boys and girls also found emotional support outside their own families, they tended to have at least one and usually several close friends, especially the girls. They relied on informal networks of kin and neighbours, peers and elders for counsel and support at the times of crisis. Some had a favourite teacher who had become a role model, friend and confidant for them [7]. Some research suggests that resilient adolescents may be in a better position to avoid risky behaviours, such as violence, substance use, and adolescent pregnancy, than are other adolescents. Adolescents who participated in evidence-based resilience-building programs, particularly those that also involve parents, showed decreases in problems with anger and aggression, in levels of perceived stress, in susceptibility to peer pressure, and in alcohol and illicit drug use, compared with adolescents who did not participated in such programs [8].

Children's behaviour also is guided in some degree by emotions. Emotions are present in every activity and they are prime movers of thought and conduct. They play an important role in influencing physical, mental health, social life, character, learning process and area of adjustment. The concept of stable emotional behaviour at any level is that which reflects the fruits of the normal emotional development. Therefore, emotional stability is considered as one of the important aspects of human life. Emotional stability is the process in which the personality is continuously striving for greater sense of emotional health, both intraphysically and intra-personally. Emotional stable individuals are calm and happy, they are satisfied with their life; they deal with the situation in perfect way and solve their problems easily [9]. An emotionally stable person has the attributes of emotional maturity, self-confidence, and stability in their plans and affections; they look boldly ahead for facts and situations and do not give into occasional fluctuations in their mood [10].

Emotional pressure is increasing day by day at adolescent stage. Emotions are basic primeval forces by nature to enable the organism to cope up with circumstances which demand the utmost effort for survival. The emotions are a way of acting, as a way of getting along in the world; they may be constructive and destructive. Emotions have strong link with urges, needs and interests. A healthy emotional development cultivates in emotional maturity. Emotional maturity is the product of interaction between many factors like home environment, school environment, society, culture and to a great extent on the programmes watched on television. Emotionally matured person can make better adjustment with himself as well as with others [11]. 
The purpose of this research was to investigate the relationship between Resilience, Emotional Stability and Perceived Stress among adolescent boys and girls studying in the 10th grade in Mumbai. Hypotheses for the current study were:

- There is no relationship between the scores of resilience, perceived stress and emotional stability of the 10th graders.

- They are no gender difference in the scores of resilience, emotional stability and perceived stress.

- There is no difference in the scores of resilience, emotional stability and perceived stress of boys and girls who have single or both parents working.

\section{METHODOLOGY}

The participants were 10th graders from ICSE and SSC board from Mumbai. There were total 50 participants, 26 females and 24 males. The responses were collected manually as well as through online google forms. The manual responses were taken from the schools where students were selected randomly and were asked to complete the questionnaire in an auditorium. All the participants were well versed with English language. A consent form was taken from each participant. The time taken to fill the online form was between $10-15$ minutes. And the time allotted for the manual response was 20 minutes all together for all the 3 scales. Manually 32 forms were collected, and 18 respondents answered the questionnaires online. The following scales were used to measure resilience, perceived stress and emotional maturity -

1. Resilience scale - The 25 item, Likert format, Wagnild and Young (1993) Resilience scale was used for data collection. It is self-reported summated rating scale, with responses ranging from strongly disagree (1) to strongly agree (7). Scoring and interpretation was in the following format: $25-100$ very low resilience; $101-115$ low resilience; 116 - 130 moderately low resilience; $131-145$ moderately high resilience; $145-160$ high resilience; $161-175$ very high resilience. Wagnild and Young (1993) reported reliability co-efficient of .91 for the scale and Alpha reliability co-efficient of 0.861 was established, both indicating that the resilience scale is reliable [12].

2. Perceived stress scale - The scale developed by Sheldon Cohen is a measure of the degree to which situations in one's life are appraised as stressful. Items were designed to tap how unpredictable, uncontrollable and overloaded respondents find their lives. The questions in the scale ask about feelings and thoughts during the last month. Scores are obtained by reversing responses to the four positively stated items $(4,5,7$, and 8$)$ and then summing across all scale items. A Cronbach's Alpha coefficient of 0.72 was obtained. This confirmed the remarkable internal consistency and stability of the scale through repeated measure tests $(0.93)$. The face validity and scale content were remarkable, and the KMO coefficient was 0.82 [13].

3. Emotional stability scale -The scale developed by A. S. Patel measures the individual's stability in daily life emotionally. It Consist of 15 questions. The range of score was from 1 to 10 . Its coefficients of reliability are 0.70 and 0.55 . Its coefficients of validity are 0.55 and 0.61 [14].

\section{RESULTS \& DISCUSSION}

The data was analysed by using descriptive (mean, standard deviation) and inferential statistics (t-test) based on objectives and the hypothesis.

Table 1: Correlation values of Resilience, Perceived Stress and Emotional Stability

\begin{tabular}{|l|l|l|l|}
\hline & Resilience & Emotional stability & Perceived stress \\
\hline Resilience & - & - & - \\
\hline Emotional stability & .104 & - & - \\
\hline Perceived stress & -.123 & -.206 & - \\
\hline
\end{tabular}

Table 1 shows the correlation scores between the three variables, i.e. resilience, emotional stability and perceived stress. The findings indicate a significant positive correlation between resilience and emotional 
stability $(\mathrm{r}=.104)$, which means if the emotional stability is high than the resilience is also high and viceversa. There was a significant negative correlation between resilience and perceived stress $(r=-.123)$, which means if resilience is high than the perceived stress is low and vice-versa. There was a significant negative correlation between emotional stability and perceived stress $(r=-.206)$, i.e., if the emotional stability is high than the perceived stress is low and vice-versa. Thus the data obtained was in line with the hypothesis. Study by Olivia Solomon (2013) on 162 students indicates negative relationship between perceived stress and academic achievement and resilience and positive relationship between resilience and academic achievement. It was also found that perceived stress and resilience together predicts academic achievement. Thus, low perceived stress and high resilience leads to high academic achievement [15].

Table 2: Mean, SD, t-value of Resilience, Emotional stability and Perceived stress of Boys and Girls

\begin{tabular}{|l|l|l|l|l|l|l|l|l|l|}
\hline & \multicolumn{3}{l}{ Resilience } & \multicolumn{3}{l|}{ Emotional Stability } & \multicolumn{2}{l|}{ Perceived stress } \\
\hline & $N$ & Mean & $S D$ & $N$ & Mean & $S D$ & $N$ & Mean & $S D$ \\
\hline Boys & 24 & 119.67 & 15.76 & 24 & 7.79 & 1.38 & 24 & 20.08 & 4.16 \\
\hline Girls & 26 & 120.42 & 16.07 & 26 & 7.65 & 1.85 & 26 & 19.96 & 3.27 \\
\hline t value & & 0.17 & & & -0.30 & & & -0.12 & \\
\hline p value & & 0.8674 & & & 0.7685 & & & 0.9284 & \\
\hline
\end{tabular}

Table 2 shows the Mean, Standard Deviation and the $t$ value of resilience, emotional stability and perceived stress of boys and girls. The mean scores for resilience of boys and girls was 119.67 and 120.42 , respectively. This indicate moderate levels of resilience. The $t$ value obtained indicate there was not much difference between the resilience scores among boys and girls. The mean scores for emotional stability of boys and girls was 7.79 and 7.65 , respectively. This indicates moderate levels of emotional stability. The t value obtained indicate there was no much difference in the emotional stability cores between boys and girls.

The mean scores for perceived stress of boys and girls was 20.08 ad 19.96, respectively. This also indicates moderate levels of perceived stress. The $t$ value indicates that there was no significant difference in the perceived stress scores of boys' and girls' difference. The findings for the three variables was not consistent with past studies. According to a study by Augustine et al. gender differences have been found in several studies. Female adolescents reported more sensitivity to interpersonal stressors and to those related to peer values regarding clothes and appearance [16].

Table 3: Mean, SD, t-value of Resilience, Emotional stability and Perceived stress of Boys and Girls who have single or both working parents

\begin{tabular}{|l|l|l|l|l|l|l|l|l|l|}
\hline & \multicolumn{3}{l}{ Resilience } & \multicolumn{3}{l|}{ Emotional Stability } & \multicolumn{2}{l|}{ Perceived stress } \\
\hline & $N$ & Mean & $S D$ & $N$ & Mean & $S D$ & M & Mean & $S D$ \\
\hline Single parent & 26 & 121.46 & 14.96 & 26 & 7.65 & 1.87 & 26 & 19.96 & 3.7 \\
\hline Both parent & 24 & 118.54 & 16.77 & 24 & 7.79 & 1.35 & 24 & 20.08 & 3.74 \\
\hline t- value & & 0.65 & & & -0.30 & & & -0.12 & \\
\hline p value & & 0.5185 & & & 0.7685 & & & 0.9084 & \\
\hline
\end{tabular}

The researchers investigated if parents - single or both parents - having a job would affect the three variables under study. There were 26 participants who have single parent working and 24 having both their parents working. It was assumed that children whose both parents were working will be more resilient, emotionally more stable and less stressed as compared to children with one parent working or they could be less resilient, less emotionally mature and more stressed. When both parents are working, children may have more responsibility and hence may be more efficient in coping with their problems or it could be the opposite. The mean resilience score of the $10^{\text {th }}$ graders having single and both parent working were 121.46 and 118.54 , 
respectively. For resilience, obtained t value indicate a non-significant relationship between the two groups. The same was found for perceived stress and emotional stability. Hence, the data obtained indicate that single or both parents working do not influence the variables under study.

\section{Limitations of the study}

1. Participants from English medium were chosen for study, which do not represent the general adolescent population.

2. No family history was taken prior to the study.

3. Sample is too small to generalize the findings.

\section{Implications of the study}

Past studies on resilience, perceived stress and emotional stability indicate that students should be trained to have high scores on resilience which in turn may help to be emotionally stable and deal effectively with personal stress. Schools should conduct workshops to help students deal with their resilience, emotional stability and perceived stress.

\section{CONCLUSIONS}

The analysed and interpreted findings of the study have led to the following conclusions: There is a significant positive correlation between resilience and emotional stability. There is a significant negative correlation between resilience and perceived stress and emotional stability and perceived stress. There is no significant difference in the resilience, emotional stability and perceived stress scores between boys and girls. The findings also indicate that there is no significant difference in the resilience, emotional stability and perceived stress scores between boys and girls having single or both parents working.

\section{REFERENCES}

1. Garmezy N, Masten AS, Tellegen A. The study of stress and competence in children: A building block for developmental psychopathology. Child Dev 1984;1:97-111.

2. Michael R. The promotion of Resilience in the face of Adversity. Cambridge University, New York, 2006.

3. Dhar N, Nandan D. Stress - a Public health menace of the day Combat it. National Institute of Health and Family Welfare, 2008.

4. Byrne DG, Davenport SC, Mazanov J. Profiles of adolescent stress: The development of the adolescent stress questionnaire (ASQ). J Adolesc 2007;30(3):393-416.

5. Emmy W., Ruth S. High risks children from birth to childhood. Overcoming the odds, 1992.

6. Smith BA, Wood BL. Psychological factors affecting disease activity in children and adolescents with cystic fibrosis: medical adherence as a mediator. Curr Opin Pediatr 2007;19(5):553-8.

7. Sarita S, Arti B., Pooja D. An Assessment of Perceived stress, Resilience and Mental Health of adolescents. Int J Sci Res Publn, 2013.

8. Compas BE. Promoting positive mental health during adolescence. Promoting the health of adolescents: New directions for the twenty-first century. 1993;159-79.

9. Astha M, Seema D, Mamta K. A study on social maturity of adolescent in Sultanpur city. Int J Home Sci 2017;3(2):278-83.

10. Hay I, Ashman AF. The development of adolescents' emotional stability and general self-concept: The interplay of parents, peers, and gender. Int J Disabil Dev Educ 2003;50(1):77-91.

11. Dutta J, Chetia P, Soni JC. A Comparative Study on Emotional Maturity of Secondary School Students in Lakhimpur and Sonitpur Districts of Assam. Int J Sc Res 2015;4(9):168-76.

12. Wagnild G. A review of the Resilience Scale. J Nurs Measure 2009;17(2):105-13.

13. Cohen S, Kamarck T, Mermelstein R. A global measure of perceived stress. J Health Soc Behav 1983;1:38596.

14. Patel AS. Emotional stability scale. Available online.(Free access).

15. Olivia S. Exploring the relationship between Resilience Perceived stress and Academic achievement. Manchester Metropolitan University, 2013.

16. Augustine LF, Vazir S, Rao SF, Rao MV, Laxmaiah A, Nair KM. Perceived stress, life events \& coping among higher secondary students of Hyderabad, India: A pilot study. Indian J Med Res 2011;134(1):61-8.

Acknowledgements - Nil; Funding - Nil; Conflict of Interest - Nil 